Research Article

\title{
Classification of left and right hand motor imagery EEG signals by using deep neural networks
}

\author{
Nuri KORHAN ${ }^{a} *$ (D) Leyla ABíLZADE ${ }^{a}$ (D), Taner ÖLMEZ ${ }^{a}$ (D, Zümray Dokur ÖLMEZ ${ }^{a}$
}

${ }^{a}$ Istanbul Technical University, Department of Electronics and Communication Engineering, Turkey

\begin{tabular}{|c|c|}
\hline ARTICLE INFO & ABSTRACT \\
\hline $\begin{array}{l}\text { Article history: } \\
\text { Received } 13 \text { September } 2021 \\
\text { Accepted } 4 \text { October } 2021 \\
\text { Keywords: } \\
\text { Common Spatial Patterns } \\
\text { Convolutional Neural Network } \\
\text { Deep Learning } \\
\text { EEG } \\
\text { Motor imagery }\end{array}$ & $\begin{array}{l}\text { The brain-computer interface (BCI) is one of the most promising technologies that allows us to } \\
\text { establish a relationship between brain and devices. In this study, three-channel EEG signals } \\
\text { collected from nine subjects performing two motor imagery tasks are classified using two different } \\
\text { deep neural network (DNN) based approaches called framework } 1 \text { (FW1) and framework } 2 \text { (FW2). } \\
\text { The proposed frameworks were evaluated using BCI Competition IV-IIb dataset. In FW1, the raw } \\
\text { EEG data is directly presented to the deep neural network without performing any pre-processing. } \\
\text { In FW2, the EEG data is first filtered with five band pass filters with fifth order (Butterworth), } \\
\text { then the common spatial patterns (CSP) method, which introduces additional pseudo channels, is } \\
\text { applied to the filtered signals. Two experiments were conducted for each framework. In the first } \\
\text { experiment, a unique DNN is trained for each subject, and in the second experiment only one DNN } \\
\text { is trained with the combination of training sets of all subjects. The performance of the two } \\
\text { experiments are then compared in terms of average accuracy. According to the simulation results, } \\
\text { we did not observe a significant difference between the average classification accuracies obtained } \\
\text { with the first and the second experiments. Therefore, we concluded that, by the use of DNNs we } \\
\text { do not need to train several subject-specific networks which requires high computational loads. } \\
\text { On the other hand, we observed that the average classification performance significantly improves } \\
\text { by the filtering and extracting features with CSP pre-processes. }\end{array}$ \\
\hline
\end{tabular}

\section{Introduction}

For decades, human brain has been studied for various purposes. These purposes span from extracting information from human brain by using brain imaging techniques [1] to transmitting the information into another environment in order to accomplish a given task [2]. Now it is known that by placing electrodes on the human scalp noninvasively, traces of the electrical activity in the brain can be observed and also can be classified by using commonly known classifiers if the features are successfully extracted by well-known signal processing techniques. Motor imagery (MI) signals, together with event related potentials (ERP) are the only signals that have been proven to work efficiently on BCI (brain computer interface) tasks [3]. Researchers have developed toolboxes and libraries in Python such as Gumpy, MNE, Wyrm in order to make it easy to process the corresponding signals [4]-[6]. However, these tools are still not enough compact and easy to use. Therefore, MATLAB and Python are used together in most cases in order to employ BCI models.

In [7], Zhang et al. compared an algorithm that contains Morlet wavelet transformation (MWT) and neural nets with CNN (convolutional neural network) by using BCI competition II dataset III which contains 280 trials that are obtained during the MI task of right and left hand. They have concluded that WNN's computational efficiency is limited. Therefore, CNN performs better. In [8], Jun yang and his co-workers have combined CNN, Discrete Wavelet Transformation, and RNN (recurrent neural network) in their study that intends to uncover the patterns 
of different EEG tasks. In their experiment, EEG recordings of six subjects were used for classifying MI (left hand) and MI (Right Foot). Recordings of seven subjects were used for classifying MI (left hand) and MI (Right Hand). They also classified the samples of MI tasks in which the imaginations of left hand and tongue were the two different tasks. This experiment is carried out with twelve subjects. They have concluded that RNN (LSTM) combined with DWT and CNN is a relatively more accurate classifier than $\mathrm{CNN}$ alone and more capable of handling subject independency in multi-task BCI applications.

In [9], Kumar et al. by using the dataset IVa of BCI competition III (five subjects, 140trials for each of the left and right hands) tried to reveal the patterns via autoencoders and then evaluated the performance of the network. They have minimized the maximum error while keeping the network computationally efficient by using RBM (restricted boltzmann machine) in combination with CSP.

In [10], Jin Zhang et al. have transformed first ten seconds of motor imagery signals into images by utilizing STFT (short time Fourier transform) and compared performances of the activation functions with a $\mathrm{CNN}$ model that contains seven layers. According to their study, SELU (scaled exponential linear unit) performs better than ELU (exponential linear unit) and RELU (rectified linear unit). Having noted that SELU works better with STFT, it is not proven to perform better than RELU in the cases where STFT is not used as a pre-processing method.

Huijuan Yang et al. have combined $\mathrm{CNN}$ and a technique called ACSP (augmented CSP) that is created by exploiting FBCSP (filter bank common spatial pattern) and Wide Band CSP (4-30HZ) in [11]. The purpose of this approach was to obtain as many features as possible and eliminate the ones of the least importance in the CNN structure so that the feature selection process would be automated. This approach has been more accurate in classifying some subjects. However, in terms of average accuracy, it did not reach the desired level of success in comparison to FBCSP.

In [12], Xiang Zhang et al. employed convolutional recurrent neural network and an auto-encoder for classifying Physionet database that is consisted of trials from ten different subjects whose motor imagery tasks are imagination of left hand, right hand, both hands and both feet. This network had considerable success in classifying Physionet database with a $95.53 \%$ maximum accuracy. However, this scenario needs to be repeated with all subjects because subject independency and generalization ability is of a crucial importance in BCI.

In this study two main frameworks, FW1 and FW2, are created in order to observe and understand the type of the change in the success of the networks and discuss how to create more accurate systems in the interpretation of BCI.
Dataset is taken from a publicly available BCI competition (BCI IV dataset IIb). This dataset consists of nine subjects that imagined to move their right hands and left hands during the trials that have been repeated 280 times in the experiment.

In the first framework the raw EEGs are fed into the network that consists of four (and five in one subject) convolutional layers and one fully connected network (FCN). The raw EEGs of each subject have been split into train and test sets, and they are fed into the networks of their own. Then, raw EEGs of all subjects are fed into the same network. The purpose of this procedure was to evaluate the inter-subject pattern dependency of the framework. It demonstrates the strength of the network in extracting different features from different sources of EEG signals.

In the second framework, the raw EEG is fed into the network that consists of four convolutional layers and one FCN. In the name of exploiting the information in different frequencies the signals are subjected to five different bandpass filters before they are fed into CSP and their corresponding features are extracted. The outputs of CSP filters are connected to a network that is identical to the network which is used in the first experiment. Just as the first framework the data of each subject is split into two parts as training and test datasets. Nine identical classifiers are evaluated separately, each having its own success rate in classifying motor imagery tasks. Finally, instead of feeding training sets one by one, all training sets are put together and used for training another network that has the same number of parameters. Then, the performance of the network is evaluated separately for each subject

\section{Methodology}

\subsection{Structure of Deep Neural Network}

Architecture of the brain computer system is shown in Figure 1. In this study, we used a DNN architecture that comprises of convolutional layers, batch normalization layers, activation layers and a FCN. The CNN extracts unique features across multiple layers to learn how to differentiate between data classes of BCI.

At first, the input is convolved with a number of filters in order to extract some features. Then it is passed through an appropriate activation function in order to make the convergence easier and faster. Finally, the information is fed into fully connected layers whose weights are determined by back propagation and used in fitting the input to the output. For the activation function, ReLU is used in this study. ReLU is the most widely used activation function for deep neural networks that removes negative pixels in the activation map and sets them to zero:

$$
f(x)=\max (0, x)
$$

There are several advantages of ReLU being more efficient and providing higher accuracy compared to other activation functions. Then every activated neuron is passed 


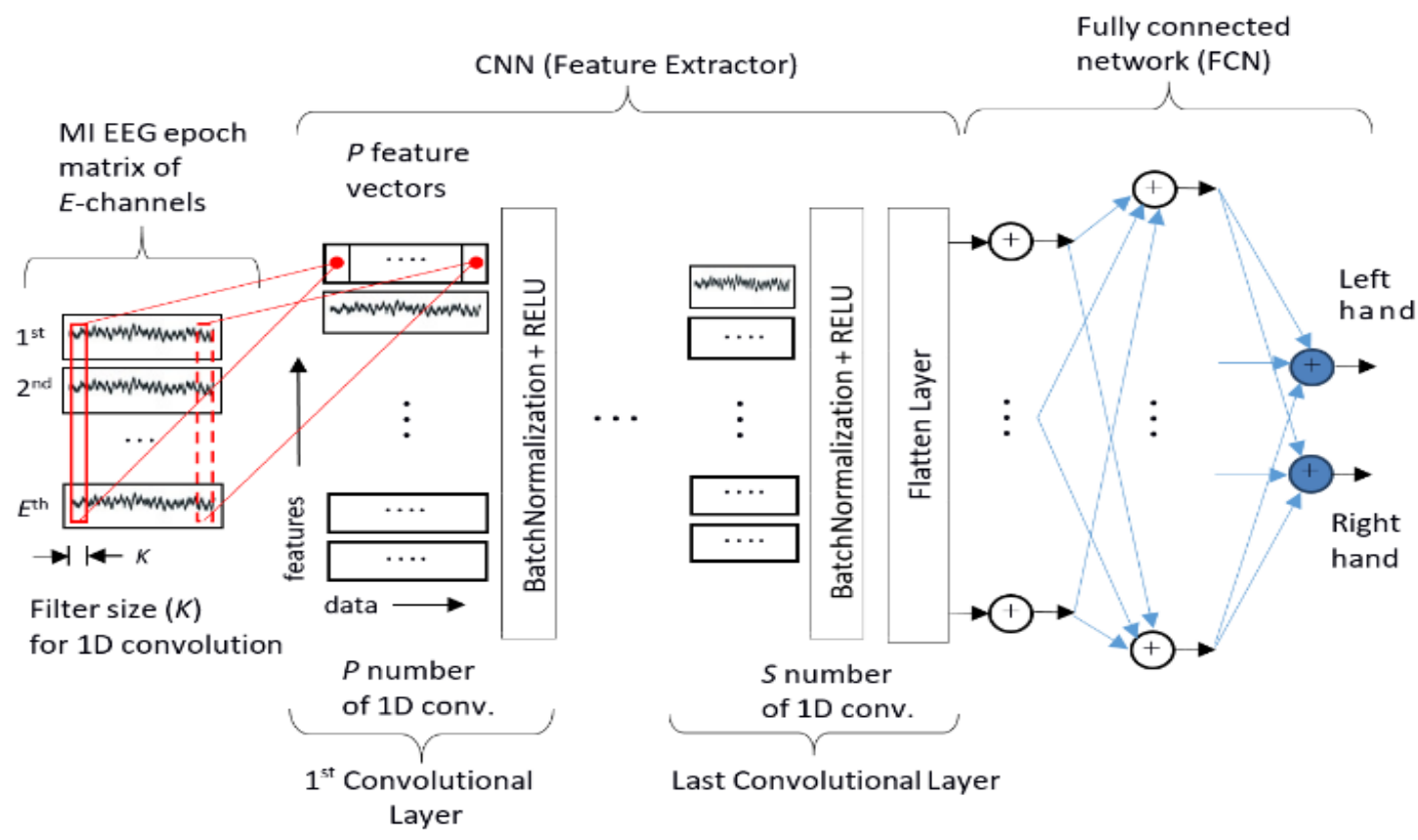

Figure 1. Architecture of the proposed DNN. E (E=3) represents the number of the channels.

to the following layer. Final stage of the DNN is the fully connected neural network which enables data to be classified. In this study, each epoch consists of 3 channels and 750 samples in each channel. The input size of the FCN is determined by the number of nodes in the flatten layer. Since the DNN of each subject is different, the number of nodes in the flatten layer is different. FCN contains one hidden layer with 1024 nodes. Since this study is dealing with a 2-class problem, there are 2 nodes at the output of the FCN. Neurons in a fully connected layer make connections with all neurons in the previous layer.

\subsection{Common Spatial Patterns as Pre-processing Stage}

The CSP is known as a powerful pre-processing tool in extracting features of EEG signals. It can efficiently distinguish different classes by maximizing variance of one class while minimizing the variance of the other class. The CSP algorithm works by diagonalization of covariance matrices that is described in the following equation the equation 2 :

$$
R_{i}=\frac{W^{T} X_{i} X_{i}^{T} W}{W^{T} X_{j} X_{j}^{T} W}
$$

where $\mathrm{X}_{\mathrm{i}}$ denotes the pre-processed signal matrix with dimensions $E \times N\left(\mathrm{X}_{\mathrm{i}} \in \mathrm{R}^{\left(E^{\times} N\right)}\right)$ in other words an epoch, where $\mathrm{i}$ is the epoch number per class, $E$ is the number of channels and $N$ is the number of samples per channel. $\mathrm{X}^{\mathrm{T}}$ is the transpose of $\mathrm{X}$ and trace function computes the sum of the diagonal elements of the matrix. In this study, EEG data from 3 channels $(\mathrm{C} 3, \mathrm{Cz}$ and $\mathrm{C} 4)$ are analyzed. In fact, CSP was used to increase the number of EEG channels artificially.

\subsection{Classification process without pre-processing stage (Framework 1)}

Two different frameworks are used and their classification performance are investigated. In the first framework, the raw data was used as input to the DNN that consists of convolutional layers that are responsible for the feature extraction and one FCN as the classification stage.

Normally the expectation would be that if the network is trained enough, it learns to recognize patterns without using any pre-processing method. The FW1 does not contain any frequency and spatial filtering process. In this case, the input of the $\mathrm{CNN}$ is the epoch matrix of dimensions $E \times N$. Tables 1 and 2 give the classification performances and the structure of the DNNs used, respectively. $3 \times N$ epoch matrices are entered into all of the DNNs shown in Table 2. ( $\mathrm{N}$ represents data size).

\subsection{Classification process using filter and CSP in pre- processing stage (Framework 2)}

In framework 2, input data is pre-processed by the method of band-pass filtering and CSP in order to increase the number of channels by creating additional pseudo channels. The output of CSP is applied to the CNN (features extracted after the CSP are given as the input to the classifier) and it is followed by a classification process via FCN. Here, the CSP helps to project the three-channel EEG data onto high-dimensional space to provide the classifier to discriminate between classes easily. A block diagram in Figure 2 represents the approach of framework 2. Tables 3 and 4 give the classification performances and the structure of the DNNs used, respectively. $2 M \times N$ epoch matrices are entered into the DNNs shown in Table 2. $(N$ and $M$ represents data size and the number of pseudo channels, respectively). It is observed in the layer1 of the Table 4 that the $M$ value is different for each subject.

Both FW1 and FW2 are trained with training sets of each of the nine subjects as well as the combination of training sets. Calculated performance results show that there is no significant difference between the average classification accuracies obtained with the first and the second experiments, i.e., results of both experiments were 
similar within each framework. However, when it comes to comparing the performance of the first and the second framework it has been observed that framework 2 provides better performance than framework 1 regardless of the preparation method of their training and test datasets.

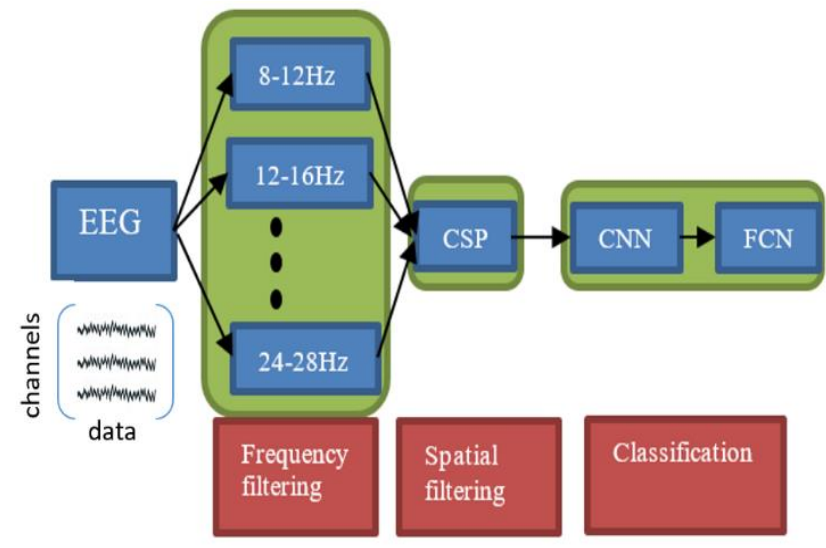

Figure 2. Classification process with pre-processing stage

\section{Experimental results}

\subsection{BCI Competition IV dataset IIb}

In this study, we used the EEG data that presented in BCI Competition Dataset IIb [13]. The dataset comprises of two classes of MI tasks (left hand and right hand) recorded from nine subjects on two separate (different) days. In total five sessions were provided per subject, including three training and two evaluation sessions. We worked only with the first two sessions of training part that consist of 240 trials without feedback in total (120 trials per session, 60 trials per class). Here each trial begins with a fixation cross and a short acoustic tone $(1 \mathrm{kHz}, 70 \mathrm{~ms})$ to prepare a subject to focus on the following command to be displayed on the monitor. At time $\mathrm{t}=3 \mathrm{~s}$ a cue in the form of arrow appear pointing to the left or right on the screen to guide the subject to execute the corresponding MI tasks of left hand and right hand respectively till $\mathrm{t}=7 \mathrm{~s}$. Afterward a trial continues with a break that lasts $1.5 \mathrm{~s}$. The paradigm for one trial is illustrated in Figure 3.

The data for each session is collected over bilaterally arranged three bipolar channels (C3, Cz and C4) according to the 10/20 system. Figure 4 shows positions of C3, C4 and $\mathrm{Cz}$ electrodes (indicated by ellipses) according to the international 10-20 system.
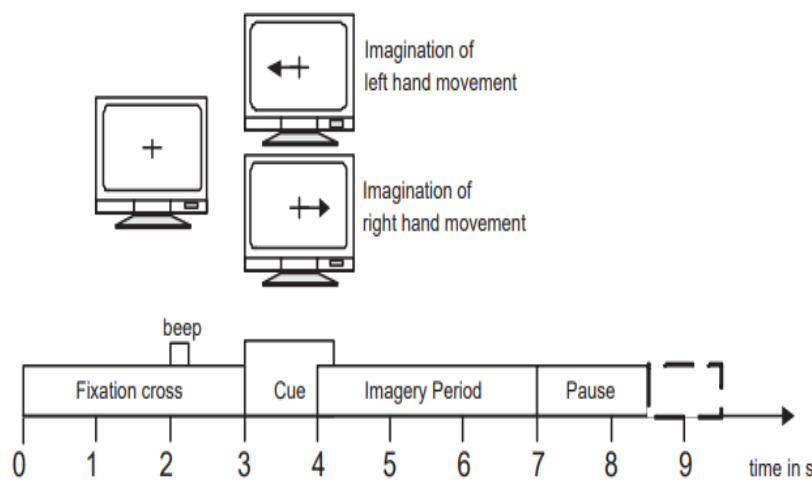

Figure 3. Architecture of the brain computer system [13]

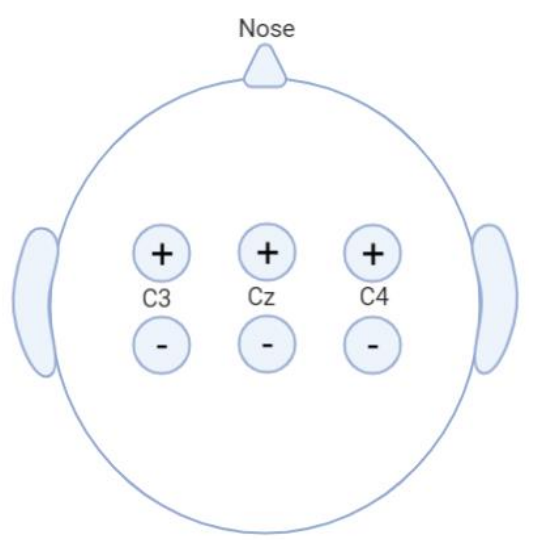

Figure 4. Positions of $\mathrm{C} 3, \mathrm{C} 4$ and $\mathrm{Cz}$ electrodes.

\subsection{Framework 1}

Table 1 shows subject-specific accuracy values for framework 1. As seen in the table, minimum test accuracy of $48.2 \%$ is obtained for Subject 6 while maximum test accuracy of $87.6 \%$ is obtained for Subject 4 . The average performance achieved is $61.2 \%$. The learning rate for all DNNs was selected as 0.0001 . Table 2 shows the size of DNNs for each subject. It is clearly seen in the table that, the size of DNN for each subject-specific dataset is small.

Table 1. Success rates of each subject for framework 1

\begin{tabular}{|c|c|c|}
\hline \multirow{2}{*}{ Subjects } & Training accuracy, \% & Test accuracy, \% \\
\hline s1 & 100 & 60.2 \\
\hline s2 & 100 & 59 \\
\hline s3 & 100 & 55.7 \\
\hline s4 & 100 & 87.6 \\
\hline s5 & 100 & 58.1 \\
\hline s6 & 100 & 48.2 \\
\hline s7 8 & 100 & 61.2 \\
\hline s9 & 100 & 60 \\
\hline
\end{tabular}

Table 2. Size of convolutional layers, filters, and feature plane for each subject in $\mathrm{FW} 1(s j=j$ th subject, $i=$ number of inputs, $f=$ size of filter, $o=$ number of outputs)

\begin{tabular}{|c|c|c|l|l|c|}
\hline & $\begin{array}{c}\text { Layer1 } \\
i, f, o\end{array}$ & $\begin{array}{c}\text { Layer2 } \\
i, f, o\end{array}$ & $\begin{array}{c}\text { Layer3 } \\
i, f, o\end{array}$ & $\begin{array}{c}\text { Layer4 } \\
i, f, o\end{array}$ & $\begin{array}{c}\text { Layer5 } \\
i, f, o\end{array}$ \\
\hline$s 1$ & $3,5,40$ & $40,5,40$ & $40,5,40$ & $40,5,40$ & NA \\
\hline$s 2$ & $3,18,20$ & $20,18,20$ & $20,18,20$ & $20,18,20$ & NA \\
\hline$s 3$ & $3,15,40$ & $40,15,40$ & $40,15,40$ & $40,15,40$ & $40,15,40$ \\
\hline$s 4$ & $3,15,20$ & $20,15,20$ & $20,15,20$ & $20,15,20$ & NA \\
\hline$s 5$ & $3,15,20$ & $20,15,20$ & $20,15,20$ & $20,15,20$ & NA \\
\hline$s 6$ & $3,15,20$ & $20,15,20$ & $20,15,20$ & $20,15,20$ & NA \\
\hline$s 7$ & $3,15,20$ & $20,15,20$ & $20,15,20$ & $20,15,20$ & NA \\
\hline$s 8$ & $3,15,20$ & $20,15,20$ & $20,15,20$ & $20,15,20$ & NA \\
\hline$s 9$ & $3,18,17$ & $17,18,17$ & $17,18,17$ & $17,18,17$ & NA \\
\hline
\end{tabular}

\subsection{Framework 2}

The proposed approach of the second framework is demonstrated in Figure 2. The learning rate for all DNNs was selected as 0.0001. Also, Table 3 shows subjectspecific accuracy values for framework 2 . The minimum test accuracy for this framework $(61.9 \%)$ is obtained for 
Subject-2 while maximum test accuracy (94.2\%) is obtained for Subject- 4 . The average performance achieved in this framework is $72.4 \%$.

Table 4 shows the size of DNNs for each subject. It is clearly seen that, the size of DNN for each subject-specific dataset is small. Paying attention to the input sizes in both Table 2 and Table 4, we see that while input sizes in Table 2 are all equal to 3, they take different values in Table 4. The number of channels at the output of the CSP transform is controlled by $2 \times M$. The number of $M$ is determined according to the subject in order to increase the classification performance and is chosen differently for each subject.

\subsection{Generalization ability of the proposed frameworks}

At first, the dataset is formed for FW1 by combining all nine datasets collected from nine subjects. In the same way, the second dataset for FW2 is created. The purpose of this arrangement is to observe the dependency of the network to the subject specific datasets. Table 5 shows the comparison of the proposed methods with the subjectspecific datasets. The structure of FW2 contains $10(M=5)$ and 49 features in the input and the other layers, respectively. The filter size for FW2 is 14 .

Table 3. Success rates of each subject for framework 2

\begin{tabular}{|c|c|c|}
\hline Subjects & Training accuracy, $\%$ & Test accuracy, $\%$ \\
\hline s1 & 100 & 79.1 \\
\hline s2 & 100 & 61.9 \\
\hline s3 & 100 & 63.2 \\
\hline s4 & 100 & 94.2 \\
\hline s5 & 100 & 72.5 \\
\hline s6 & 100 & 82.9 \\
\hline s7 & 100 & 66 \\
\hline s8 & 100 & 72.3 \\
\hline s9 & 100 & 65 \\
\hline
\end{tabular}

Table 4. Size of convolutional layers, filters, and feature plane for each subject in $\mathrm{fw} 2$ ( $s j=j$ th subject, $i=$ number of inputs, $f=$ size of filter, $o=$ number of outputs)

\begin{tabular}{|c|c|c|c|c|}
\hline & $\begin{array}{c}\text { Layer1 } \\
i, f, o\end{array}$ & $\begin{array}{c}\text { Layer2 } \\
i, f, o\end{array}$ & $\begin{array}{c}\text { Layer3 } \\
i, f, o\end{array}$ & $\begin{array}{c}\text { Layer4 } \\
i, f, o\end{array}$ \\
\hline$s 1$ & $20,23,20$ & $20,23,20$ & $20,23,20$ & $20,23,20$ \\
\hline$s 2$ & $6,13,20$ & $20,13,20$ & $20,13,20$ & $20,13,20$ \\
\hline$s 3$ & $2,13,20$ & $20,13,20$ & $20,13,20$ & $20,13,20$ \\
\hline$s 4$ & $2,13,30$ & $30,13,30$ & $30,13,30$ & $30,13,30$ \\
\hline$s 5$ & $10,13,30$ & $30,13,30$ & $30,13,30$ & $30,13,30$ \\
\hline$s 6$ & $2,13,20$ & $20,13,20$ & $20,13,20$ & $20,13,20$ \\
\hline$s 7$ & $2,13,30$ & $30,13,30$ & $30,13,30$ & $30,13,30$ \\
\hline$s 8$ & $14,3,30$ & $30,2,30$ & $30,2,30$ & $30,2,30$ \\
\hline$s 9$ & $10,23,20$ & $20,23,20$ & $20,23,20$ & $20,23,20$ \\
\hline
\end{tabular}

Table 5. Generalized comparison of fw 1 and fw2

\begin{tabular}{|l|c|c|}
\hline & $\begin{array}{c}\text { Mean accuracy of } \\
\text { the combined } \\
\text { datasets, } \%\end{array}$ & $\begin{array}{c}\text { Mean accuracy } \\
\text { of the subject } \\
\text { specific } \\
\text { datasets, } \%\end{array}$ \\
\hline Training Accuracy & 100 & 100 \\
\hline Test Accuracy by FW1 & 64.3 & 61.2 \\
\hline Test Accuracy by FW2 & 76 & 74 \\
\hline
\end{tabular}

Table 6 shows the classification results of MI EEG signals obtained by using different feature extraction methods and classifiers. The results shown in this study were compared with studies in the literature using the same dataset. In this study, the performance results were obtained independent of the subject.

Table 6: The classification of MI EEG signals by the neural networks with different topologies.

\begin{tabular}{|l|l|l|l|l|}
\hline methods & $\begin{array}{c}\text { accuracy } \\
\%\end{array}$ & $\begin{array}{l}\text { transformation } \\
\text { or } \\
\text { preprocessing }\end{array}$ & classifier & $\begin{array}{c}\text { BCI } \\
\text { datasets }\end{array}$ \\
\hline In [15] & 77.6 & STFT & DNN & IV - IIb \\
\hline In [16] & 62.5 & Bispectrum & SVM & IV - IIb \\
\hline In [17] & 67.8 & $\begin{array}{l}\text { Linear } \\
\text { Predictive } \\
\text { Coding }\end{array}$ & MLP & IV - IIb \\
\hline In [18] & 77.5 & $\begin{array}{l}\text { Filter + } \\
\text { normalization }\end{array}$ & DNN & IV - IIb \\
\hline In [19] & 76.4 & LSTM & DNN & IV - IIb \\
\hline $\begin{array}{l}\text { In our } \\
\text { study }\end{array}$ & 78 & CSP & DNN & IV - IIb \\
\hline
\end{tabular}

LSTM: Long-short Term Memory

Figure 5 and Figure 6 demonstrate the spatial filter visualization obtained for left and right hands by using combined dataset, respectively. In Figures 5 and 6, white color shows higher activations. It is observed that the activations for the left and right hands occur in the appropriate positions of the brain.

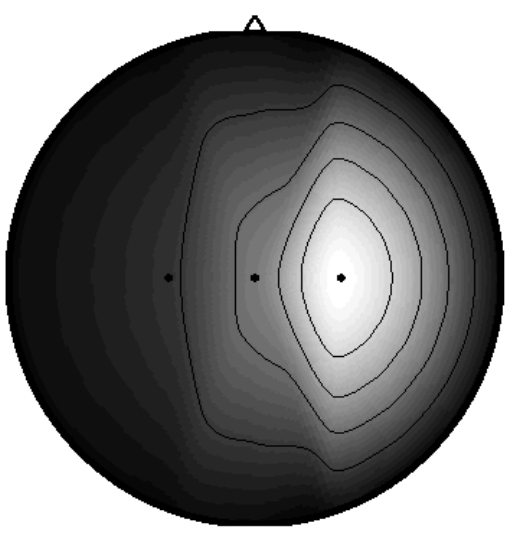

Figure 5. Spatial filter visualization for the left hand.

\section{Conclusion}

In this study, the impact of the number of channels on the classification performance has been investigated. FW2 is employed in order to increase the number of channels virtually. This process has been carried out by using filters and the important channels are then selected by utilizing CSP method. However, it is known that the CSP suffers from subject specificity severely. Therefore, DNN is proposed in order to eliminate that disadvantage of CSP.

As seen in Table 1, FW1 has not been successful enough 
in classifying EEG-MI signals by exploiting only three channels. It is clearly seen in Table 2 that increasing the number of channels has increased the test accuracy by ten percent.

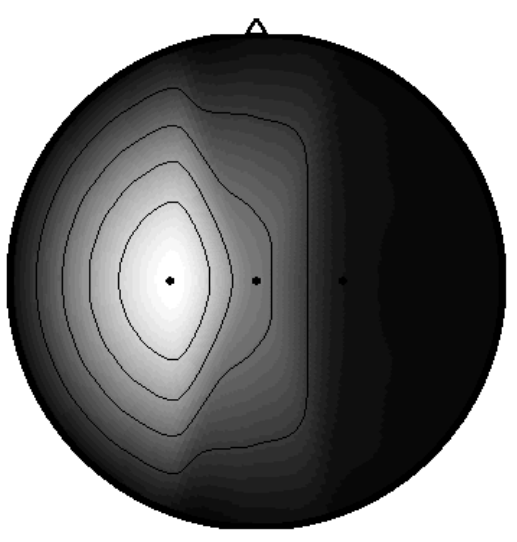

Figure 6. Spatial filter visualization for the right hand.

As predicted, the disadvantage of CSP is eliminated by using DNN as seen in Table 5. In the studies of Dai [14] and Tabar [15], 78.2\% and $77.6 \%$ classification performances were obtained for BCI Competition Dataset IIb, respectively. In these studies, researchers used datasets with feedback. Therefore, their success rate is higher thanks to using these datasets. The focus of our study is to investigate the effects of channel number on classification performance.

The CNN used for the analysis of all EEG signals of the dataset has six convolutional layers. At the output of each convolutional layer, there are batch normalization and ReLU layers. The size (N) of input signal is 750 . For all convolutional layers, the sizes of filters are 17 , and the numbers of all feature planes are 40 . In this setup, $78 \%$ classification performance is achieved for the MI BCI Competition Dataset IIb.

\section{Acknowledgment}

This work was supported by the Istanbul Technical University Scientific Research Project Unit [ITU-BAP MYL-2018-41621].

\section{References}

[1] D. Tan and A. Nijholt, "Brain-computer interfaces and humancomputer interaction," in Brain-Computer Interfaces, Springer, pp. 3-19, 2010.

[2] K. LaFleur, K. Cassady, A. Doud, K. Shades, E. Rogin, and B. $\mathrm{He}$, "Quadcopter control in three-dimensional space using a noninvasive motor imagery-based brain-computer interface," J. Neural Eng., vol. 10, no. 4, p. 46003, 2013.

[3] F. Lotte and I. B. Sud-ouest, "BCI's Beyond Medical Applications.pdf," pp. 26-34, 2012.

[4] Z. Tayeb et al., "Gumpy: A Python toolbox suitable for hybrid brain-computer interfaces," J. Neural Eng., vol. 15, no. 6, p. 65003, 2018.

[5] B. Venthur, S. Dähne, J. Höhne, H. Heller, and B. Blankertz,
"Wyrm: A brain-computer interface toolbox in python," Neuroinformatics, vol. 13, no. 4, pp. 471-486, 2015.

[6] A. Gramfort et al., "MNE software for processing MEG and EEG data," Neuroimage, vol. 86, pp. 446-460, 2014.

[7] Z. Zhang et al., "A Novel Deep Learning Approach With Data Augmentation to Classify Motor Imagery Signals," IEEE Access, vol. 7, pp. 15945-15954, 2019.

[8] J. Yang, S. Yao, and J. Wang, "Deep Fusion Feature Learning Network for MI-EEG Classification," IEEE Access, vol. 6, pp. 79050-79059, 2018.

[9] S. Kumar, A. Sharma, K. Mamun, and T. Tsunoda, "A deep learning approach for motor imagery EEG signal classification," in 2016 3rd Asia-Pacific World Cong. on Com. Sci. and Eng. pp. 34-39, 2016.

[10] J. Zhang, C. Yan, and X. Gong, "Deep convolutional neural network for decoding motor imagery based brain computer interface," in 2017 IEEE Int. Conf. on Sig. Pro., Comm. and Comp., pp. 1-5, 2017.

[11] H. Yang, S. Sakhavi, K. K. Ang, and C. Guan, "On the use of convolutional neural networks and augmented CSP features for multi-class motor imagery of EEG signals classification," Proc. Annu. Int. Conf. IEEE Eng. Med. Biol. Soc. EMBS, pp. 26202623, 2015.

[12] X. Zhang, L. Yao, Q. Z. Sheng, S. S. Kanhere, T. Gu, and D. Zhang, "Converting your thoughts to texts: Enabling brain typing via deep feature learning of eeg signals," in 2018 IEEE Int. Conf. on Perv. Comp. and Comm., pp. 1-10, 2018.

[13] M. Tangermann et al., "Review of the BCI competition IV," Front. Neurosci., vol. 6, p. 55, 2012.

[14] M. Dai, D. Zheng, R. Na, S. Wang, and S. Zhang, "EEG Classification of Motor Imagery Using a Novel Deep Learning Framework," Sensors, vol. 19, no. 3, p. 551, 2019.

[15] Y. R. Tabar and U. Halici, "A novel deep learning approach for classification of EEG motor imagery signals," J. Neural Eng., vol. 14, no. 1, p. 16003, 2016.

[16] N. Kotoky and S.M. Hazarika, "Bispectrum Analysis of EEG for Motor Imagery Classification", Int., conf. on Sig. Pro. and Integ. Netw., pp. 581-586, 2014.

[17] V.F. Silva, R.M. Barbosa, P.M. Vieira, C.S. Lima, Ensemble learning based classification for BCI applications, IEEE 5th Portuguese Meeting on Bioengineering, (2017).

[18] D.M. Hermosilla, R.T. Codorniú, R.L. Baracaldo et al., Shallow Convolutional Network Excel for Classifying Motor Imagery EEG in BCI Applications, IEEE Access, 9, 98275-98286, 2021.

[19] B. Yang, C. Fan, C. Guan, X. Gu and M. Zheng, "A Framework on Optimization Strategy for EEG Motor Imagery Recognition", 41st Ann. Int. Conf. of the IEEE Eng. in Med. and Bio. Soc., pp. 774-777, 2019. 